Jūlija Zālīte

ORCID: 0000-0001-7632-0228

Universität Lettlands

DOI: $10.19195 / 0435-5865.144 .20$

\title{
Wie realisiert eine Talkshow-Prominenz den Sprecherwechsel in unterschiedlichen Medienkulturen? Ein Vergleich politischer Talkshows in Deutschland und Lettland
}

\begin{abstract}
s
Das Ziel dieses Beitrags ist es, exemplarisch aufzuzeigen, wie die Gesprächsteilnehmer mit einer großen Erfahrung vor der Kamera den Sprecherwechsel in zwei unterschiedlichen Medienkulturen realisieren. Als Grundlage für diese Untersuchung dienen zwei lettische Sendungen des politischen Talkshowformates („Kas notiek Latvija?“, „Sastrēgumstunda“) und zwei deutsche politische Fernsehtalkshows („Hartaberfair“, „Maybrit Illner“). Für die Detailanalyse wurden Textbeispiele ausgewählt, die den Versuch das Rederecht zu verteidigen oder den Kampf um das Rederecht im Gespräch aufweisen. In diesen Gesprächspassagen wurde der Sprecherwechsel nicht nur auf der verbalen, sondern auch auf der nonverbalen und prosodischen Ebene analysiert. Die Untersuchungsergebnisse haben aufgezeigt, dass die Gesprächsteilnehmer mit einer großen Erfahrung vor der Kamera in beiden Ländern unterschiedliche Wege wählen, um den Sprecherwechsel zu realisieren. In den deutschen politischen Fernsehtalkshows sind zwischen dem Moderator und der Talkshow-Prominenz eindeutige Versuche der Unterbrechung und reale Unterbrechungen festzustellen. Die lettische Talkshow-Prominenz präferiert bei der Realisierung ihrer Turns ohne expressive Gestik zu kommunizieren und wenn eine Überlappung mit dem Moderator entsteht, zieht sie sich eher aus dem Gespräch ohne ihre eigene Konstruktion abzuschließen.
\end{abstract}

Schlüsselwörter: Gesprächsanalyse, Sprecherwechsel, Polit-Talkshows

\section{How do professional speakers implement turn-taking in different media cultures? Conversational analysis of Latvian and German political talk shows}

This paper aims to examine how professional speakers implement turn-taking in different media cultures. Two Latvian ("Kas notiek Latvijā?", "Sastrēgumstunda") and two German ("Hartaberfair", 
"Maybrit Illner") political talk shows were chosen and considered as the materials of the study. The text samples for detailed analysis are selected according to the following principle: during a discussion with other speakers, a professional speaker tries to take or hold the floor. In these samples, the turn-taking system is analyzed not only on a verbal but also on a nonverbal and prosodic level. Results show that professional speakers in Latvian political talk shows and in German political talk shows prefer different strategies to get the right to talk. Between moderators and professional German speakers, clear attempts of interruption and real interruptions are determined. Conversely, the professional Latvian speakers prefer to communicate without expressive gestures and, if there is an overlap with the moderator, they tend to withdraw from the conversation without completing their own construction.

Keywords: conversational analysis, turn-taking, political talk shows

Jūlija Zālīte, Latvijas Universitāte, Humanitāro zinātņu fakultāte, Visvalža iela 4a, Rīga, LV-1050, Lettland, E-Mail: julijakokina@gmail.com

Received: 7.10.2018, accepted: 8.04.2019

\section{Vorbemerkungen}

Heutzutage ist die Forschungsrichtung „Gesprochene Sprache“ ein etablierter Wissenschaftsbereich in der Linguistik und es besteht kein Zweifel, dass die gesprochene Sprache ihre Besonderheiten, ihr eigenes System hat und dass die Grammatik der gesprochenen Sprache zu untersuchen ist (vgl. Auer 2010, Hennig 2006). Die Beschreibungskategorien der schriftsprachlichen Texte können nur selten in Dienst gestellt werden, um den gesprochenen Text zu beschreiben. Nachdem die Technik es erlaubt hat, die mündliche Kommunikation aufzunehmen und präzise Tanskripte bereitzustellen, ist Mitte der 1960er Jahre ein Bedarf nach neuen Kategorien enstanden. Die Sprachwissenschaftler beschäftigen sich seitdem intensiv mit gesprochener Sprache und beschreiben die Elemente, die für die Analyse relevant sind. Eines der wichtigsten Elemente der mündlichen Kommunikation stellt der Sprecherwechsel dar. Ähnlich wie in der Morphologie ist das Morphem eine Beschreibungskategorie, so ist der Sprecherwechsel in der gesprochenen Sprache eine Grundeinheit (vgl. Sacks/Schegloff/Jefferson 1974). Der Sprecherwechsel tritt besonders häufig in den politischen Fernsehtalkshows in Erscheinung. Die politischen Fernsehtalkshows sind nicht selten auf Konfrontation gerichtet und dies prägt die Systematik des Sprecherwechsels wesentlich. Aber auch die medialen Aspekte (z.B. Unterhaltung und Wunsch nach positiver Selbstdarstellung u.a.) machen die Untersuchung von Sprecherwechseln besonders relevant und erforderlich. Dass die Erforschung von Sprecherwechseln ${ }^{1}$ ihre Aktualität nicht

${ }^{1}$ Im deutschsprachigen Raum hat bereits im Jahre 1975 Ulrike Geißner in ihrem „Gesprächsverlaufssoziogramm“ (1975) nicht nur formal-strukturelle Aspekte des Sprecherwechsels mit einbezogen, wie das der Fall im Aufsatz von Sacks et al. (1974) war, sie protokollierte bereits nonverbale Aspekte und verfolgte die Rolle des Blickkontakts auf den Verlauf der Gesprächsstruktur. 
verloren hat, bestätigen die gegenwärtigen multimodalen Untersuchungen ${ }^{2}$, die neue Erkenntnisse hervorgebracht haben. Im Rahmen der multimodalen Perspektive wurde festgestellt, dass nicht nur das Hörbare bei der Realisierung des Sprecherwechsels beteiligt ist, also nicht nur die Teilnehmer, die verbal aktiv sind, sondern alle Gesprächsteilnehmer ${ }^{3}$ auch mit ihrer Gestik, ihrer Nonverbalität an dem Zustandekommen des Sprecherwechsels mitwirken (vgl. Deppermann/Schmitt 2007: 22).

\section{Forschungsziel, Forschungsmethoden, Textkorpus}

Das Ziel dieser Untersuchung ist es, anhand von ausgewählten Transkripten der deutschen und lettischen politischen Talkhsows exemplarisch aufzuzeigen, wie die Gesprächsteilnehmer mit einem hohen Bekanntheitsgrad und einer großen Erfahrung vor der Kamera den Sprecherwechsel ${ }^{4}$ in einer so komplizierten Ge-

${ }^{2}, \mathrm{Zu}$ den unterschiedlichen Dimensionen oder Modalitätsebenen, die bei der Interaktionskonstitution eine Rolle spielen und die in der grundsätzlichen Körperlichkeit und Raumgebundenheit der ,Inter-Aktion' basieren, gehören beispielweise: Verbalität (Prosodie inbegriffen) und ihre Körperlichkeit; Vokalität; Blick; Kopfbewegung; Mimik; Gestikulation; Körperpositur; Bewegungsmodus; Präsenzform; Proxemik: Verteilung im Raum: Positionierung der Beteiligten (face to face, side by side, back to face...), Nähe/Distanz Räumlichkeit/Materialität: Manipulation von Objekten (Tools, Instrumente etc.). Aus einer multimodalen Perspektive auf Interaktion werden die unterschiedlichen Dimensionen oder Modalitätsebenen theoretisch als grundsätzlich gleichwertig konzipiert. Keine der Dimensionen ist demnach für die Analyse der Interaktionssituation zentraler oder wichtiger als andere: Blickkontakt oder Körperpositur kommen als Modalität theoretisch, methodisch und konzeptuell der gleiche Status zu wie der Verbalität. Konkret tritt eine Modalität nur dann in den Vordegrund, wenn sie von den Beteilgten selbst zur situationssensitiven Realisierung eines bestimmten Handlungszusammenhangs erkennbar relevant gesetzt wird“ (Mondada/Schmitt 2007: 24 f.).

3 Joanna Pędzisz in ihrem Aufsatz ,Zur Rollendynamik in dem Kommunikationsgefüge politische Talkshow“ kommt zu ähnlichen Schlussfolgerungen: „In der Analyse eines Gesprächs in der politischen Talkshow dürfen also nicht nur die Perspektive des Sprechers und seine verbalen und nonverbalen Handlungen berücksichtigt werden. Die Aktivitäten des Hörers, der auf die kommunikativen Handlungen des Sprechers reagiert, machen das Wesen eines Gesprächs aus, das zum interaktiven Phänomen wird“ (Pędzisz 2007: 70).

${ }^{4}$ In der klassischen Definition wird unter Sprecherwechsel ein Übergang des Rederechts vom Sprecher an den Hörer verstanden (vgl. etwa Duncan/Fiske 1977, Rath 1979, Brinker 2010). Dabei wird auf der verbalen Handlungsebene von einer Sprecher-Hörer Konstellation ausgegangen „The turn system [...] requires each participant to consider himself to be either a speaker or an auditor at each moment in the interaction. At each such moment a speaker is a participant who claims the speaking turn. An auditor is a participant who does not claim the speaking turn at a given moment. These two postulated states are considered to be discrete ans, within each participant, mutually exclusive. The speaker and auditor states are clearly classifications each participant applies to himself and can known to others only through his actions“" (Duncan/Fiske 1977: 177) .

Wie aus dieser Definition zu lesen ist, dominiert an dieser Stelle die Verbalität und die Rolle des Sprechers im Gespräch lässt sich als aktiv bezeichnen, die Rolle des Hörers als passiv. Die non- 
sprächsform wie der kontroversen Debatte realisieren und ob kulturspezifische Unterschiede sichtbar werden. Die Fokussierung auf den Sprecherwechsel ist in dieser Arbeit einerseits dadurch zu erklären, dass er als zentraler Punkt der Organisation des Gesprächs gilt und gleichzeitig ein wichtiges Mittel zur Zweckrealisierung der einzelnen Beteiligten ist. Beim Sprecherwechsel geht es weniger um den Redebeitrag. Im Zentrum der Aufmerksamkeit steht die Art und Weise, wie die Gesprächsteilnehmer in der Diskussion zu Wort kommen, was eng mit der Rolle des Sprechers zusammenhängt (z.B. hat ein Moderator in der Polit-Talkshow eine privilegierte Rolle und er hat das Recht, ggf. den Gast zu unterbrechen). Andererseits hat eine andere Beobachtung den Impuls zu dieser Untersuchung gegeben. In den Talkshows ist eine Steigerung der Interaktionsdynamik durch Regelverstöße von Gesprächsbeteiligten festzustellen. Dazu gehören u.a. der Kampf um das Rederecht mit Unterbrechungen, Ausdehnung des eigenen Redebeitrags, die Missachtung von Regeln der zivilisierten Redeübergabe sowie Redeüberlappungsturbulenzen.

Als Grundlage für die folgende Untersuchung dienen vier Sendungen des politischen Talkshowformats „Hartaberfair“ und „Kas notiek Latvija?“5, die dem Thema Finanzkrise 2008 gewidmet sind. Die anderen zwei, „Maybrit Illner” und die lettische Talkshow „Sastrēgumstunda“, behandeln das immer noch aktuelle Thema der Flüchtlinge im Mittelmeer. Für eine Detailanalyse wurden alle Sendungen zuerst grob und nur einzelne Textpassagen wurden nach dem GAT-Verfahren transkribiert. Das aufgenommene und transkribierte Videomaterial für die Analyse umfasst 322 Minuten. Die Untersuchung orientiert sich im wesentlichen an den methodischen Ansätzen der Gesprächsanalyse, die zur materialgestützten qualitativen Forschungsdisziplin gehört.

\section{Exemplarische Analyse}

In allen vier analysierten Fernsehtalkshows dominiert das Gespräch zwischen der Talkshow-Prominenz und dem Moderator. Deshalb wurden für die weitere Detailanalyse Textbeispiele in Situationen ausgewählt, wie Talkshow-Prominenz während der Diskussion mit dem Moderator oder wie Talkshow-Prominenz versucht, mit anderen prominenten Gästen das Wort zu ergreifen oder zu behalten. Die Detailanalyse von ausgewählten Textpassagen fand statt um zu erfahren, welches Verhalten von den Talkshowgästen in solchen Situationen beim Sprecherwechsel präferiert wird. Die nachstehenden Gesprächsausschnitte wurden als typische Beispiele für die Kommunikation unter der Talkshow-Prominenz und

verbale Handlungsebene mit der Berücksichtigung solcher Ausdrucksmodalitäten wie z.B. proxemische Distanzierung wird nicht in Betracht gezogen, wie das in multimodalen Untersuchungen der Fall ist.

${ }^{5}$ Diese politische Talkshow ist 2011 aus dem Fernsehprogramm genommen worden. 
dem Moderator herangezogen. Dabei wurde nicht nur die verbale Ebene der Kommunikation in Betracht gezogen, sondern auch die nonverbale Ebene. Die Untersuchung von nonverbaler Kommunikation wurde nur an den Stellen zur Analyse herangezogen, an denen die Gestik für das Erreichen des kommunikativen Ziels besonders erfolgreich war.

(1) Das folgende zur Analyse stehende Beispiel (45:59-47:12) stammt aus der politischen Talkshow „Hartaberfair”. In diesem Gesprächsausschnitt sind drei Talkshow-Prominente und der Moderator beteiligt. Der Ausschnitt liegt relativ spät im Gespräch, die Gäste diskutieren bereits seit vierzig Minuten miteinander. Vor dieser Passage lief ein Einspieler, in dem behauptet wurde, dass eine abfällige Haltung gegenüber der Wissenschaft bis heute in der Politik salonfähig sei. Dieser Fall ist deshalb interessant und relevant, weil der Moderator in dieser Passage eindeutig zeigt, dass er ein Problem mit der Platzierung der Äußerungsstarts der anderen Teilnehmer hat. Die prominenten Gäste dagegen lassen sich vom Moderator nicht steuern.

Direkt nach dem Einspieler ergreift der Moderator Frank Plasberg das Wort, wendet sich mit einer Anrede BL (Bernd Lucke) zu und stellt ihm eine Frage:

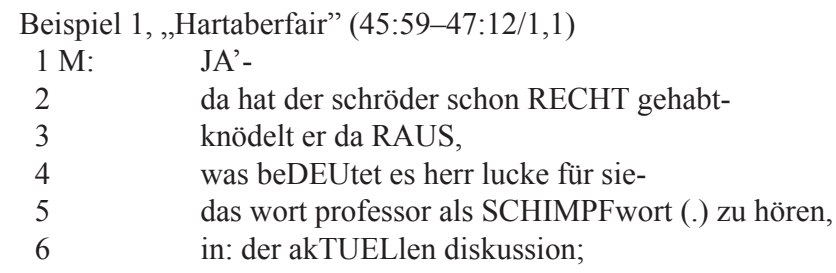

Während der Moderator seinen Turn produziert, lächelt BL ihm zu. Direkt nachdem der Moderator seinen Beitrag beendet hat, geht BL nach einem glatten Sprecherwechsel direkt auf die Frage des Moderators ein (Zeile 7-21). In seiner Äußerung unternimmt BL aber einen Vergleich (Zeile 19-21), mit dem MF (Michel Friedman) nicht einverstanden ist (Zeile 22).

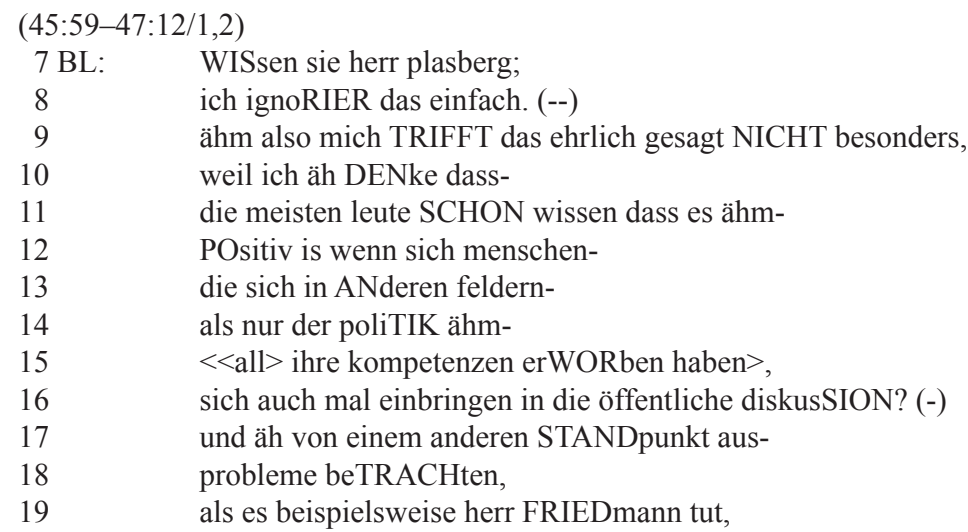


20 der alles immer unter dem poLItischen aspekt sieht,

21 und die [wirtschaft]lichen realitäten dann neGIERT,

$22 \mathrm{MF}: \quad[<<\mathrm{p}>$ nein $>]$

Noch während der Artikulation von BLs Äußerung immer unter dem poLItischen aspekt sieht, und die [wirtschaft]lichen realitäten dann neGIERT, beginnt MF verneinend seinen Kopf zu schütteln. Gleichzeitig zieht er seine Augenbrauen nach oben, als wäre er über die Äußerung BLs sehr erstaunt, und dann reagiert MF auf die Aussage BLs mit einem leisen nein als Zeichen dafür, dass er mit dessen Behauptung nicht einverstanden ist. Im Anschluss an die Konstruktion BLs übernimmt der Moderator das Rederecht und möchte es an KGE (Katrin GöringEckardt) weitergeben. Nach der Namensnennung frau göring ECKardt macht der Moderator eine kurze Pause ${ }^{6}$, die BL für den Start seines Turns nutzt (Zeile 24).

$(45: 59-47: 12 / 1,3)$

$23 \mathrm{M}$ : $\quad$ frau göring ECKardt;

24 BL: jetzt (.) [MÖCHte ich noch-]

$25 \mathrm{M}$ : [frau göring ECKardt;

26 CL: (ich) hab VIEL von Ökonomie [gesprochen herr professor.]

$27 \mathrm{BL}$ : [DAgegen,]

28

29 nein (.) $\mathrm{d}=$ die arguMENte,

$<<$ all $>$ die sie erWÄHNT haben;

30 habe ich überHAUPT=nicht bei ihnen gehört muss ich sagen.>

(Mehrere Teilnehmer reden durcheinander.)

BL schafft nur ein Wort ungestört zu realisieren, jetzt, denn der Rest der Konstruktion wird gleichzeitig mit dem sofortigen Einsatz des Moderators realisiert (Zeile 24, 25). Dieser versucht, zum zweiten Mal, gleichzeitig zu der Äußerung BLs mit einer Anrede, MÖCHte ich noch, KGE das Wort zu erteilen. Der Einsatz BLs ist ein deutlicher Versuch, das Rederecht zu erobern, obwohl der Moderator die Redeübergabe an KGE bereits durchgeführt hat. An dieser Stelle steigt der dritte Gast CL in das Gespräch ein, wendet sich an BL (Zeile 26) und ergänzt das von ihm aufgegriffene Thema. BL wartet nicht, bis CL mit seiner Konstruktion fertig ist, sondern beginnt simultan zu gesprochen herr professor eine Negierung: DAgegen, nein=die arguMENte, $<<$ all $>$ die sie erWÄHNT haben; habe ich überHAUPT =nicht bei ihnen gehört muss ich sagen. Es folgt eine unverständliche Passage über ein paar Sekunden, denn mehr als drei Gesprächsteilnehmer reden durcheinander. Im Anschluss an diese Turbulenzphase ist die Konstruktion KGEs zu hören, die simultan zu einem Einsatz von CL produziert wird.

An dieser Stelle soll daran erinnert werden, dass KGE vorher bereits zweimal vom Moderator zur Redeübernahme angeregt wurde. Auch dieses Mal schafft KGE es nicht, ihr Rederecht in dem Grad zu übernehmen, wie es der Moderator eingeplant hatte (das beweist der weitere Turnübergabeversuch des Moderators,

${ }^{6}$, ,Hitches ${ }^{6}$ generate recipient activities. And not infrequently the activity engendered by a ,hitch' is the starting up of a recipient's talk, regardless of the (in)auspiciousness or interactional (im) propriety of starting at such a place“" (Jefferson 1983: 27). 
Zeile 34), sie schafft es nur, einen kurzen Kommentar abzugeben. Der Rückzug KGEs aus der Sprecherrolle bedeutet nicht nur die Vermeidung einer potenziellen Rivalität um die Redegelegenheit (Zeile 33), sondern sie beendet das simultane Sprechen, weil sonst beide Gefahr laufen können, sich umsonst zu artikulieren. CL wird aber sofort vom Moderator aufgehalten, und durch den Einsatz nee nee nee nee nee jetzt frau göring ECKardt äußert der Moderator noch simultan zu CL eine Konstruktion. Er streckt seine Hand in die Richtung KGEs, um auch nonverbal deutlich zu machen, dass sie jetzt das Rederecht erteilt bekommen hat. CL lässt sich nicht unterbrechen und führt seine Äußerung weiter (Zeile 36). Der Moderator reagiert darauf, indem er sich in Richtung CL zu bewegen beginnt. Als der Moderator CL schon erreicht hat, ergreift er ihn mit der Hand, und versucht mit einer konkreten und lauten Anrede HALlo herr LINdner, dessen Redefluss zu hemmen.

Dieser Aufruf wird simultan zu der Konstruktion CLs realisiert (Zeile 37). Aber auch dieses Mal ignoriert CL den verbalen und nonverbalen Versuch des Moderators, das Rederecht an eine andere Gesprächspartnerin überzuleiten, und führt seinen Einsatz weiter (Zeile 38-40). Nun bewegt sich der Moderator in die Richtung KGEs, um allen anzuzeigen, dass sie die nächste Sprecherin sein soll (Zeile 39-41).

$\begin{array}{ll}\text { (45:59-47:12/1,4) } \\ 31 \mathrm{KGE}: & \text { [dagegen ist ja vielleicht nichts zu SAgen.] } \\ 32 \mathrm{CL}: & {[<<>\text { (nur vielleicht) in erINnerung rufen- }} \\ 33 & \text { dass (herr) LUcke }>\text { ] } \\ 34 \mathrm{M}: & \text { [<<all> nee nee nee nee nee, }> \\ 35 & \text { jetzt frau göring ECKardt; } \\ 36 \mathrm{CL}: & \text { !EIN! [professor ist, }=\text { ] } \\ 37 \mathrm{M}: & \text { [HALlo herr LINdner.] } \\ 38 \mathrm{CL}: & \text { =aber nicht !DIE! volkswirtschaft; } \\ 39 & \text { es gibt nämlich auch ANdere professoren, } \\ 40 & \text { [die ANdere meinungen haben als sie.] } \\ 41 \mathrm{BL}: & \text { [ziemlich viele davon sind unserer] parTEI herr lindner. } \\ 42 \mathrm{KGE:} & \text { [ALso.] } \\ 43 & \text { ah ja. } \\ 44 & \text { vi=viele davon sind in ihrer parTEI herr lu:cke=; } \\ 45 & <<\text { all }>=\text { und äh GLEICHzeitig muss man ja vielleicht KLAR und deutlich sa- } \\ & \text { gen-> } \\ 46 & \text { dass wir !AUCH! deswegen (--) in der situation= } \\ 47 & \text { =auch in der WIRTschaftlichen situation in euROpa sind? } \\ 48 & \text { weil es immer !GANZ! viele ExPERten gab, } \\ 49 & \text { profesSOrinnen und profesSOren- } \\ 50 & \text { die gesagt haben auf JEden fall muss man SO machen; } \\ 51 & \text { man muss alles machen wie man=s traditionell IMmer gemacht hat; }\end{array}$

Währenddessen unternimmt CL keinen Rückzug aus der Sprecherrolle, sondern führt seinen Gedanken zu Ende (Zeile 40). Noch innerhalb des laufenden Turns von CL initiiert BL die Äußerung ziemlich viele davon sind unserer parTEI herr lindner, die anscheinend nicht warten kann. KGE wartet nicht, bis BL seine Konstruktion beendet hat, und mit einem lauten ALso gibt sie den anderen Gästen 
zu verstehen, dass sie auf paralleles Sprechen eingestellt ist und um die Sprecherrolle konkurriert. Endlich kann KGE sich als neue Sprecherin etablieren.

(2) In den lettischen politischen Talkshows konnte nur ein Beispiel gefunden werden, in dem die Talkshow-Prominenz ins Gespräch mit dem Moderator kommt und in dem die Talkshow-Prominenz bereit war, ihre Sprecherrolle zu verteidigen. Dieses Beispiel wurde zur Analyse aus der Sendung „Sastrēgumstunda“ entnommen, weil der Rest der Daten entweder einen glatten Sprecherwechsel zwischen der Talkshow-Prominenz und dem Talkshow-Laien aufgezeigt haben oder der Einsatz vom Moderator zu einem Abbruch des Gesprächsbeitrags führte.

Die nachstehende Gesprächspassage entwickelt sich zu dem Zeitpunkt, als die Sendung beinahe eine Viertelstunde im Gange ist. Im Mittelpunkt dieses Gesprächs steht die Frage, was die Bevölkerung Lettlands nicht akzeptieren kann, die Quoten oder die Flüchtlinge selbst. Am analysierten Dialog sind die Prominenz RZ (Roberts Zīle) und der Moderator beteiligt. Der Moderator hat vorher die Frage an RZ gestellt, was er zu diesem Thema denkt und die abgebildete Sequenz zeigt, wie sich das Gespräch weiter entwickelt. Als außergewöhnlich, mit Blick auf die restlichen Daten der lettischen Sendungen, stechen die Aktivitäten der Prominenz $\mathrm{RZ}$ in den Zeilen 15 und 16 hervor.

Bevor RZ seine Äußerung in einer Frageform initiiert (Zeile 1 und 2) erklärt er, dass die Billigung der Flüchtlings-Quoten keine Gefahr für Lettland sei. Obwohl die Quoten 20000 Flüchtlinge voraussetzen, ändert diese Zahl in der Realität nichts, denn Lettland nimmt sowieso viel mehr Flüchtlinge auf, als diese Quoten es vorschreiben. In der Zeile 1 formuliert RZ seine Äußerung in einer Frageform und nach einer sehr kurzen Pause beantwortet er diese Frage selber (Zeile 3):

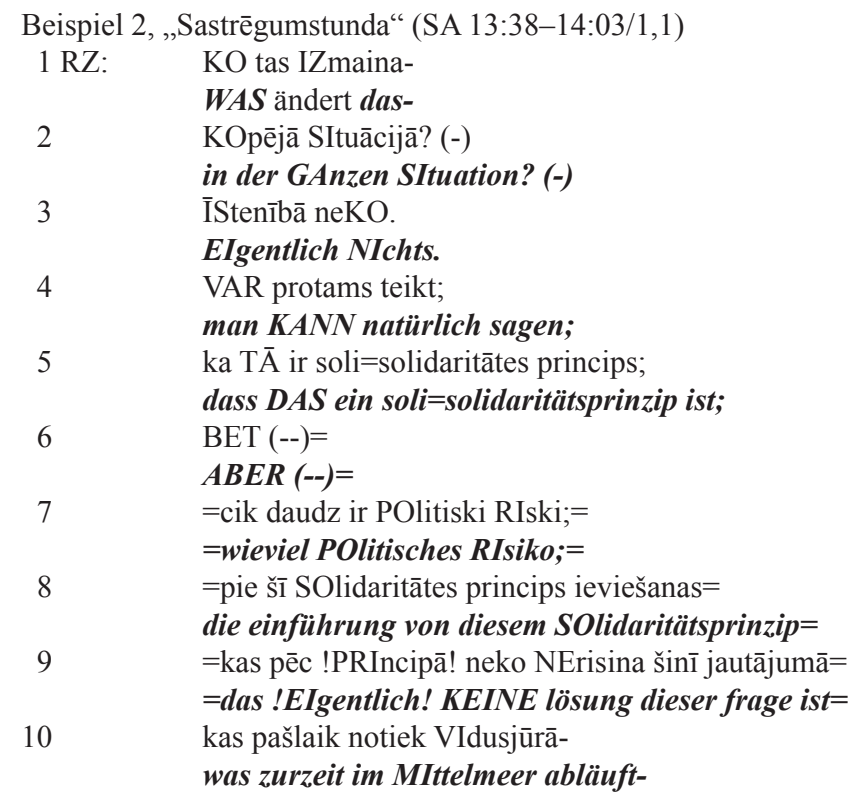


Dann fügt er einen weiteren Aspekt bei und behauptet, dass dieses Solidaritätsprinzip die Situation mit den Flüchtlingen im Meer nicht ändern wird, aber für Lettland könnte dies ein politisches Risiko werden. Den Redebeitrag produziert RZ in einem ruhigen Tempo und ohne expressive Gestik. Während der Realisierung seiner Äußerung schaut RZ den Moderator an. Auch die Blickrichtung von anderen Gästen ist hauptsächlich auf den Moderator zentriert. Die Lautstärke während der Produktion des Redebeitrags bleibt gleich, nur das akzentuierte Wort principā wird etwas lauter realisiert. An manchen Stellen hat RZ Formulierungsschwierigkeiten (Zeile 5 und 9).

In der Zeile 12 setzt der Moderator ein. Der Start ist nicht laut gestaltet, nur das Wort ir ist stark akzentuiert, um die Entgegensetzung zu markieren. Der Moderator macht in seiner Äußerung deutlich, dass die Billigung der Quoten in Lettland den Flüchtlingen eine Hilfe leisten würde. Er stimmt RZ nicht zu, dass die Hoffnungen nicht erfüllt werden können. Einen Moment lang sprechen RZ und der Moderator simultan, denn der Moderator hat seine Aussage nicht am Ende der von RZ realisierten Einheit gestartet (Zeile 11), sondern an einer nicht-übergaberelevanten Stelle. RZ setzt seine Ausführungen fort, der Einstieg des Moderators führt nicht zum Abbruch der ursprünglichen Äußerung. In den Zeilen 15 und 16 fordert RZ, ihn weiter fortfahren zu lassen. RZ bearbeitet diese Störung mit der Bemerkung $n \bar{e}, n u$ pagaidiet. Mit dem Wort $n \overline{\mathrm{e}}$ und der Äußerung $n u$, pagaidiet drückt RZ die Unabgeschlossenheit seiner Aussage aus. Die akzentuierte Partikel $n \bar{e}$ drückt in diesem Fall nicht eine verneinende Antwort aus, sondern mit der Partikel $n u$ zusammen, wird eine Warnung (vgl. Smiltniece 2013: 682) ausgedrückt. Er zeigt damit, dass er die Äußerung noch zu Ende formulieren möchte und sich nicht aus der Sprecherrolle zurückziehen wird.

(SA 13:38-14:03/1,2)

$13 \mathrm{M}: \quad[<<$ all $>$ bet tas tomēr IR I < all $>$ aber das IST

14 solis uz PRIEkšu $>$; [ein schritt WEITer>];

$15 \mathrm{RZ}: \quad \mathrm{NE}$;

NEIN;

16 nu: PAgaidiet; na: WArten sie;

17 nu šie CILvēki na: diese LEUte -

18 DIVsimt pār CILvēkiüber ZWEI hundert MEnschen -

19 kas BŪs; die KOmmen; 
20

21

21

22

23

24

$((\ldots))$ vini atradīs DArbu;

sie finden einen $\mathrm{JOB}$;

PIEnemsim;

NEHmen wir an;

sāks STRĀdāt;

fangen an zu ARbeiten;

viņi jebKUrā brīdī -

sie JEDERzeit -

var braukt TUR-

\section{können fahren DORThin-}

kur viņi VĒlās.

wohin sie WOllen.

Aus dem weiteren Verlauf des Dialogs ist zu entnehmen, dass der Moderator diesen Hinweis wahrnimmt und erlaubt dem Gast seinen Beitrag abzuschließen.

\section{Resümee}

Vergleicht man die Kommunikation zwischen dem Moderator und der Talkshow-Prominenz in den deutschen und in den lettischen analysierten politischen Talkshows, so lassen sich einige Unterschiede feststellen. Die prominenten Talkshowgäste in den deutschen politischen Fernsehtalkshows kommunizieren aktiv miteinander und mit dem Moderator und das nicht nur verbal, sondern auch nonverbal. Zwischen dem Moderator und der Talkshow-Prominenz sind eindeutige Versuche der Unterbrechung und reale Unterbrechungen festzustellen. Mithilfe der Konstruktionswiederholungen, heftigen Bewegungen mit den Armen und Händen, Akzentuierungen, lautem Sprechen, gelingt es der Talkshow-Prominenz nicht nur ihr Rederecht zu verteidigen, sondern sie verhindert drei Mal die Redeverteilung des Moderators, sodass der Moderator sich aus der Sprecherrolle zurückziehen muss. An den Stellen, wo ein simultanes Sprechen zwischen dem Moderator und der Talkshow-Prominenz entsteht, bricht der Gast seine Konstruktion nicht ab, er führt sein Redezug zu Ende, denn nicht selten ist der prominente Gast schon vorher auf paralleles Sprechen eingestellt. Die nachstehende Tabelle bietet einen Überblick darüber, wie die Talkshow-Prominenz auf der verbalen, nonverbalen und prosodischen Ebene ihre Sprecherwechsel mit dem Moderator in den analysierten

Textpassagen gestalten: 
Tab. 1: Sprecherwechselrealisierung der deutschen und lettischen Talkshow-Prominenz im Gespräch mit dem Moderator

\begin{tabular}{|c|c|c|c|c|c|}
\hline & & Verbal & Prosodisch & Nonverbal & Sonstiges \\
\hline 1. & $\begin{array}{l}\text { Talkshow-Pro- } \\
\text { minenz } \rightarrow \\
\text { Moderator } \\
\text { (deutsche } \\
\text { politische Talk- } \\
\text { shows) }\end{array}$ & $\begin{array}{c}\text { Zu Wort } \\
\text { kommen } \\
\text { meistens über } \\
\text { die Selbstwahl; } \\
\text { Überlappungen; } \\
\text { lange Redebei- } \\
\text { träge; Struk- } \\
\text { turierung von } \\
\text { Redebeiträgen; } \\
\text { längeres simul- } \\
\text { tanes Sprechen; } \\
\text { Turbulenzpha- } \\
\text { sen; explizite } \\
\text { Redewünsche; } \\
\text { gescheiterte } \\
\text { Redeübernah- } \\
\text { meversuche des } \\
\text { Moderators; } \\
\text { Wortabbrüche, } \\
\text { unbeendete } \\
\text { Redeeinheiten; } \\
\text { Wiederho- } \\
\text { lungen. }\end{array}$ & $\begin{array}{c}\text { Akzentuierte } \\
\text { Wörter und } \\
\text { Silben; Anstieg } \\
\text { der Lautstärke; } \\
\text { Anstieg der } \\
\text { Sprech-ge- } \\
\text { schwin-digkeit; } \\
\text { lautes Sprechen. }\end{array}$ & $\begin{array}{c}\text { Heftige Be- } \\
\text { wegungen mit } \\
\text { den Armen und } \\
\text { Händen. }\end{array}$ & $\begin{array}{c}\text { Gedankenwie- } \\
\text { derholungen } \\
\text { sind nicht } \\
\text { erwünscht und } \\
\text { werden unter- } \\
\text { brochen. Der } \\
\text { Talkshow-Pro- } \\
\text { minente startet } \\
\text { nicht selten sei- } \\
\text { nen Redebeitrag } \\
\text { inmitten des } \\
\text { Redebeitrags } \\
\text { des Moderators. } \\
\text { Ignoranz der } \\
\text { verbalen und } \\
\text { nonverbalen Re- } \\
\text { derechts-Vertei- } \\
\text { lungs-Versuche } \\
\text { des Moderators. } \\
\text { Die promi- } \\
\text { nenten Gäste } \\
\text { lassen sich vom } \\
\text { Moderator nicht } \\
\text { steuern. Deut- } \\
\text { liche Zeichen } \\
\text { von Turnkompi- } \\
\text { tition; Turbu- } \\
\text { lenzphase kann } \\
\text { nur mithilfe des } \\
\text { Moderators auf- } \\
\text { gelöst werden. }\end{array}$ \\
\hline 2. & $\begin{array}{l}\text { Talkshow-Pro- } \\
\text { minenz } \rightarrow \\
\text { Moderator } \\
\text { (lettische } \\
\text { politische Talk- } \\
\text { shows) }\end{array}$ & $\begin{array}{l}\text { Kurzes simul- } \\
\text { tanes Sprechen; } \\
\text { Überlappungen. }\end{array}$ & $\begin{array}{c}\text { Ruhiges Tempo; } \\
\text { Akzentuierte } \\
\text { Wörter (Parti- } \\
\text { kel); gefüllte } \\
\text { Denkpausen. }\end{array}$ & $\begin{array}{l}\text { Blickricht-ung } \\
\text { auf den Modera- } \\
\text { tor zentriert. }\end{array}$ & $\begin{array}{c}\text { Zeichen von } \\
\text { Turnkompititi- } \\
\text { on. Der Einstieg } \\
\text { des Moderators } \\
\text { führt nicht zum } \\
\text { Abbruch. Die } \\
\text { Talkshow-Pro- } \\
\text { minenz bearbei- } \\
\text { tet die Störung } \\
\text { des Moderators } \\
\text { (mit Vernei- } \\
\text { nungspartikel). }\end{array}$ \\
\hline
\end{tabular}


Nach der Analyse der ausgewählten lettischen Textpassagen kann festgehalten werden, dass sich die Talkshow-Prominenz, wenn eine Überlappung mit dem Moderator entsteht, eher aus dem Gespräch zurückzieht ohne ihre eigene Konstruktion abzuschließen. Auch bei der Redeübernahme wartet sie bis der vorherige Sprecher seinen Redezug beendet hat und fängt erst dann an seine eigene Rede zu realisieren. Und nur ein Mal konnte festgestellt werden, dass der Einsatz des Moderators inmitten des Redebeitrags von der Talkshow-Prominenz nicht zum Abbruch des Turns führte. Der Gast zeigte in den analysierten Textpassagen auf der verbalen Ebene, dass er nicht bereit ist, sich aus der Sprecherrolle zurückzuziehen und verteidigte sein Recht auf das Sprechen. Die lettische Talkshow-Prominenz präferierte bei der Realisierung ihrer Turns ein ruhiges Tempo, gleichbleibende Lautstärke und kommunizierte ohne expressive Gestik.

\section{Literatur}

Auer, Peter (2010): Zum Segmentierungsproblem in der Gesprochenen Sprache. In: InLiSt - Interaction and Linguistic Structures 49, S. 7-36. Online verfügbar unter http://www.inlist.uni-bayreuth.de/issues/49/InList49.pdf (Zugriff am 11.07.2018).

Brinker, Klaus / Sager, Sven Frederik (2010): Linguistische Gesprächsanalyse. Eine Einführung. 5. Aufl. Berlin.

Deppermann, Arnulf / Schmitt, Reinhold (2007): Koordination. Zur Begründung eines neuen Forschungsgegenstandes. In: Schmitt, Reinhold (Hrsg.): Koordination. Analysen zur multimodalen Interaktion. Tübingen. S. 15-54.

Duncan, Starkey / Fiske, Donald Winslow (1977): Face-to-Face Interaction: Research, Methods, and Theory. New York.

Geißner, Ulrike (1975): Gesprächsverlaufssoziogramm. In: Geißner, Helmut K. (Hrsg.): Rhetorik und Pragmatik. Sprache und Sprechen. Bd. 5. Ratingen/Kastellaun. S. 49-82.

Hennig, Mathilde (2006): Grammatik der Gesprochenen Sprache in der Theorie und Praxis. Kassel. Online verfügbar unter https://www.mediensprache.net/archiv/pubs/3906.pdf (Zugriff am 9.08.2016).

Jefferson, Gail (1986): Notes on Some Orderlinesses of Overlap Onset. In: D’Urso, Valentina (Hrsg.): Discourse Analysis and Natural Rhetoric. Padua. S. 11-38.

Mondada, Lorenza / Schmitt, Reinhold (2008): Zur Multimodalität von Situationseröffnungen. In: Mondada, Lorenza / Schmitt, Reinhold (Hrsg.): Situationseröffnungen. Zur multimodalen Herstellung fokussierter Interaktion. Tübingen. S. 7-53.

Pędzisz, Joanna (2007): Zur Rollendynamik in dem Kommunikationsgefüge politische Talkshow. Online verfügbar unter http://bazhum.muzhp.pl/media//files/Lublin_Studies_in_Modern_Languages_and_Literature/Lublin_Studies_in_Modern_Languages_and_Literature-r2007-,t31/ Lublin_Studies_in_Modern_Languages_and_Literature-r2007-t31-s68-85/Lublin_Studies in_Modern_Languages_and_Literature-r2007-t31-s68-85.pdf (Zugriff am 18.01.2018).

Rath, Rainer (1979): Kommunikationspraxis. Analysen zur Textbildung und Textgliederung im gesprochenen Deutsch. Göttingen.

Sacks, Harvey / Schegloff, A. Emanuel / Jefferson, Gail (1974). A Simplest Systematics for the Organization of Turn Taking in Conversation. In: Language 50, S. 696-735. Online verfügbar unter https://pure.mpg.de/rest/items/item_2376846/component/file_2376845/content (Zugriff am 12.07.2018).

Smiltniece, Gunta (2013). Izsauksmes vārds. In: Latviešu valodas gramatika. Rīga. S. 679-692.

Germanica Wratislaviensia 144, 2019

(C) for this edition by CNS 